Pecvnia, 2 (2006), pp. 1-21

\title{
Una estrategia cooperativa en la pequeña empresa comercial de Castilla y León: El caso $6000^{1}$
}

\author{
Eduardo Fuentes Ganzo
}

\begin{abstract}
En los últimos tiempos estamos asistiendo, en el campo de la Dirección estratégica, a nuevas formulaciones que inciden en la importancia de focalizar las estrategias empresariales en mercados y ámbitos locales. En este sentido, el comercio tradicional en los espacios locales, constituido básicamente por empresas familiares, ha contemplado, en los últimos 15 años, cómo las fuerzas competitivas de su entorno se han visto alteradas por la irrupción generalizada de Hipermercados y grandes superficies. En el ámbito territorial de Castilla y León esta realidad ha propiciado la aparición de nuevas estrategias cooperativas entre las empresas locales de pequeño comercio. Uno de estos ensayos estratégicos locales, realizado con éxito, se estudia en este trabajo.
\end{abstract}

Palabras clave: Estrategia local, cooperación, Empresa familiar, comercio minorista, PYMEs.
In recent times, as far as the strategic management is concerned, new proposals have been suggested, which are focused on the local markets. In that sense, the traditional corner shop, basically settled an run by members of the same family have seen a massive emergence of big shopping centres for the last 15 years. This new reality has allowed the appearance of new cooperative strategies among small local firms in the region of Castilla y León (Spain). This paper analyses one of these strategic alliances that has been successful.

Key words: Local Strategy, cooperation, Family Firms, retail trade, SMEs.

1 Deseo manifestar mi agradecimiento a la Confederación zamorana de Empresarios, CEOE-CEPYME- ZAMORA, y especialmente a su Secretario General, D. Luis de Luis, y a la Confederación abulense de empresarios, CONFAES, con mención especial a D. Javier Marfull, Secretario General de la Federación de Comercio, por su colaboración y los muchos datos facilitados para este estudio. 


\section{INTRODUCCIÓN}

Todo, o casi todo, parecía dicho, desde finales de los 60, cuando se publicó El Nuevo Estado Industrial (Galbraith 1967), que suponía el reconocimiento teórico de las nuevas formas de empresa que habían emergido con el siglo, firmas concebidas como grandes organizaciones y "tecoestructuras" convertidas en los actores protagonistas del nuevo escenario global; hasta que, a pesar del reconocimiento de esa realidad, a inicios de los 70, otro economista Schumacher, tras su experiencia como gestor económico rural en cuencas carboníferas, reconoció que, con todo y a pesar de ello, "Lo pequeño es hermoso" (1973), que, a pesar de la existencia de grandes conglomerados y organizaciones empresariales, era posible implementar con éxito tecnologías intermedias que requerían poca inversión, que se podían fomentar pequeñas empresas familiares en entornos, incluso, rurales, que, en definitiva, cabía apostar por economías de escala humana, activas y dinamizadoras de las pequeñas comunidades.

Igualmente, un par de décadas después, en lo que respecta a los planteamientos estratégicos de la empresa, todo, o casi todo, parecía dicho, tras las muy sustantivas aportaciones de Porter (1980 y 1990), de Grant (1995) o de Hax y Majluf (1997), que supusieron el diseño de armazones teóricos e instrumentos, pensados fundamentalmente para la gran empresa, hasta que, recientemente, se están escuchando nuevas voces, como las de Greenwald y Kahn (2005a y 2005b) que exaltan el valor de las "pequeñas estrategias" en entornos locales más reducidos y abarcables, entornos donde imperan menos las economías de escala que ciertas barreras de entrada, entornos, en fin, mucho más limitados donde resulta más fácil mantener la consumer captivity con bajos costes.

De hecho, son estas pequeñas empresas familiares las que sostienen fundamentalmente el crecimiento y el tejido económico en muchas regiones, tal es el caso de Castilla y León, la región interior más extensa de Europa, en contacto perimetral con Comunidades españolas y portuguesas, un magnífico ej emplo de esa economía de "lo pequeño", que también impone estrategias de "lo pequeño". Espacios donde las medidas humanas de todas sus poblaciones son abarcables y próximas, constituyendo las principales poblaciones áreas y subáreas comerciales según la terminología del Atlas Comercial de España (1994), salvo, acaso, con la única excepción de Valladolid. Áreas donde su tejido económico básico es la suma de pequeñas empresas de carácter familiar, que han 
conseguido, y están consiguiendo, desarrollar actividades económicas de éxito en diversos ámbitos como el del turismo interior (Paradores o Pousadas en Portugal), el turismo rural (Casas y albergues rurales) en el sector agroalimentario (Bodegas vitivinícolas que jalonan toda la cuenca del Duero, o fabricas de quesos), con éxito razonable.

En este contexto, el pequeño comercio minorista, formado mayoritariamente por pequeñas empresas familiares, ha tenido que enfrentarse al reto de competir con la intensa implantación, desde inicios de la década de los 90, de hipermercados, cadenas y grandes superficies comerciales. El nivel de competencia que ejercen estas grandes superficies con productos que sustancialmente coinciden con los del pequeño comercio, de modo absoluto en algunos casos -alimentación, electrodomésticos- y relativo en otros -ropa, calzado, peluquería, belleza, ocio- (González Benito 2000) producen la imposibilidad, para estas pequeñas microempresas comerciales, en su mayoría de carácter familiar, de competir en costes. Esto les ha forzado a la asunción de estrategias más imaginativas, que incidan en las únicas alternativas estratégicas que les quedaban: la diferenciación y la cooperación. Su pequeño tamaño y escasa capacidad económica ha llevado a los más innovadores a la asunción de estrategias cooperativas amparadas por las organizaciones y federaciones empresariales de comercio. Una de las cuales, por su carácter modelizador y el éxito obtenido va a ser objeto de nuestro análisis. Siendo utilizada con resultado en diversas poblaciones de Castilla y León con distintas nomenclaturas: La Campaña 6000 ó de los 6000 euros. La articulación de esta estrategia ha tenido particular incidencia en poblaciones de tamaño reducido que mantienen un carácter de centro comercial, del que depende, además del centro urbano, un conjunto de poblaciones de carácter rural para las que constituye un centro comercial comarcal. De entre ellas, sustancialmente idénticas, hemos elegido la de Benavente por la abarcabilidad de las empresas implicadas (poco más de un centenar en este caso).

Únicamente nos resta añadir que el tratamiento del que hemos denominado "Caso 6000" aunque es susceptible de ser analizado desde la muy interesante perspectiva del marketing, y por ende focalizado desde esta especialidad, nosotros hemos optado por abordarlo y reflexionar sobre el mismo desde el estricto ámbito de algunas de sus implicaciones estratégicas. 
Una estrategia cooperativa en la pequeña empresa comercial de Castilla y León: El caso 6000

\section{LA CAMPAÑA: DESCRIPCIÓN E IMPLICACIONES ESTRATÉGICAS. HIPÓTESIS DE TRABAJ 0}

La actuación consiste en la realización de una campaña de ventas navideñas, en la que se integran colectivamente un gran número de empresas de pequeño comercio de una localidad o de un determinado espacio local (por ejemplo "comerciantes de la zona centro"), generalmente adscritos a una organización o federación empresarial que los aglutina. En virtud de la misma se realiza una campaña publicitaria intensiva junto con un sorteo de 6.000 euros entre todos los compradores de los establecimientos participantes. La campaña, de un mes de duración, se inicia en noviembre, realizándose un sorteo ante notario, en vísperas de la lotería de Reyes, sorteo de gran prestigio, arraigo y tradición social en España.

La campaña, cuyo coste total oscila entre los 20.000 y los 30.000 euros $^{2}$, incluyendo el premio de los 6.000 euros, se realiza mediante inserciones publicitarias en los medios locales y provinciales (prensa local y provincial, cuñas radiofónicas y televisiones locales). El resto del capítulo presupuestario se dedica a la impresión y difusión de trípticos, octavillas, carteles y los cupones de sorteo que se entregan a los clientes.

La práctica, durante el periodo, consistía en que cada comercio participante entregaba un cupón por cada fracción de compra, por ejemplo cada 6 euros o cada 10 euros. Cupón que el cliente cumplimentaba con sus datos personales y que, a su vez, entregaba para el sorteo en el propio establecimiento o en las entidades colaboradoras (Caj as de Ahorros, ayuntamientos, asociaciones empresariales...).

Otro aspecto reseñable de esta estrategia cooperativa es que suele tener un coste "0" para las empresas participantes, ya que para la financiación se implica a la administración regional (J unta de Castilla y León a través de sus consejerías de Economía o de Fomento), al ayuntamiento de la localidad, a una entidad financiera (generalmente una Caja de Ahorros que adecua más sus fines a participar en las dotaciones de este tipo de actuaciones) y a la Cámara de Comercio que opera en la

Datos facilitados por la confederación abulense de empresarios (CONFAES) y 
zona. Instrumentalizándose la financiación a través de un convenio de Colaboración con la asociación de Empresarios que promueve la iniciativa.

Finalmente, tras el cierre de la campaña, se reúnen los cupones y se verifica, en la fecha prefijada, un sorteo ante notario al que se le da la máxima difusión en busca del impacto publicitario. Al ganador se le pone un coche a su disposición, durante un día para gastarse, entre los establecimientos participantes, el premio, con unas condiciones tasadas en las bases del sorteo como fijar un gasto máximo en cada establecimiento participante (300 euros), salvo en uno, para el que sufija un límite superior (1.200 euros).

El antecedente pionero en este tipo de campañas en Castilla y León hay que buscarlo en Ávila, concretamente en la Federación de Comercio de CONFAES (Confederación abulense de empresarios) que inició la práctica de esta estrategia en la campaña 1998-9, y que desde entonces ha realizado ininterrumpidamente una campaña anual hasta la pasada edición (8a) correspondiente al periodo navideño 2004-5. Los pequeños comerciantes abulenses, en una ciudad que apenas rebasa los 50.000 habitantes vivieron con preocupación el establecimiento en la ciudad de las grandes cadenas PRYCA y CARREFOUR (esta última con $7.000 \mathrm{~m}^{2}$ ), iniciando esta estrategia promocional diferenciadora, no desde la confrontación, sino tratando de mantener y recuperar sus espacios entre los consumidores. Desde entonces, y emulando las experiencias abulenses, se han seguido prácticas similares en otras ciudades de Castilla y León; otro ejemplo emblemático, puede ser el caso de Zamora, con características similares de entorno social y pequeño volumen demográfico, con una población de apenas 60.000 habitantes, donde la Asociación zamorana de Empresarios (CEOE-CEPYME-Zamora) a través de su Federación de Comercio (AZECO) ha acometido una iniciativa similar bajo el lema "Año-seismil-euro", desde el año 2001, realizándola, también ininterrumpidamente, por lo que en la actualidad ya ha realizado la $4^{a}$ campaña, la correspondiente al periodo 2004-5.

Evidentemente, en estos singulares casos de estrategia cooperativa, y siguiendo a Porter (1987), de entre las dos posibles alternativas estratégicas que cabe asumir por parte de las empresas: Estrategia de Costes o Estrategia de diferenciación, se opta inequívocamente por la segunda, dado el pequeño volumen y capacidad económica de las empresas implicadas, siendo notorio que los recursos y capacidades de los pequeños comercios son muy diferentes y asimétricos respecto a los grandes, por lo que han de tratar de sustituir la ventaja competitiva del 
Una estrategia cooperativa en la pequeña empresa comercial de Castilla y León: El caso 6000

rival utilizando esas habilidades diferentes (Barney 1999; Aragón Sánchez y Rubio Bañón 2005). Todo ello en el marco de lo que se ha dado en Ilamar Estrategia promocional realizada concertadamente por una asociación de comerciantes que articulan con carácter defensivo un "asociacionismo espacial" (Burnett 1997; Bigné 2003). Se trata, en consecuencia, de una clara estrategia de diferenciación articulada cooperativa y asociadamente, con una insólita ventaja adicional para este tipo de estrategias (que siempre, en sede teórica, comportan costes elevados) como es el que en el caso que nos ocupa estos costes (significativos para la capacidad económica de los participantes) son asumidos por la actuación cooperativa y por ende trasladados a las instituciones y entidades colaboradoras, con las que la asociación empresarial establece un convenio de financiación ad hoc. De esta forma queda salvado el principal obstáculo que toda estrategia de diferenciación suele conllevar, y que hace que en ocasiones, sobre todo cuando se cuenta con escasos recursos el formulador de la estrategia quede "atrapado en la mitad" dado que estas actuaciones "suelen ser caras" (Porter 1987).

El resultado de este tipo de estrategias se traslada o bien a una diferenciación en el producto físico o a prácticas destinadas a incrementar el valor añadido percibido con una clara orientación al mercado. En este caso concreto, consiste en una serie de prácticas de mercadotecnia que buscan como resultado una percepción por parte del cliente de aumento del valor del producto ante la eventualidad de un precio sustancial no competitivo con el de las grandes superficies. Independientemente de esta percepción principal, se transmiten, al cliente, otras, como las que inciden en su percepción de un mejoramiento de sus desempeños (Porter 1987) por parte de esas empresas, con mejoras que enfatizan, para los consumidores, la diferenciación y excelencia de los minoristas, el trato personalizado, la especialización, etc. Actuación que, desde otro punto de vista, puede ser percibida en términos de una estrategia defensiva de respuesta frente a las grandes superficies (Thompson y Strickland 2001).

Todo esto supone, parafraseando a Porter, la creación para esas empresas, en ese entorno local, "de un desempeño superior". Se busca la alteración premeditada y cooperativa del entorno específico que podemos visualizar utilizando el instrumento analítico de las "5 fuerzas" (Porter 1980), modelo que sigue siendo muy consistente, aunque susceptible de ser completado como hace Grant al cuestionarse sí son cinco, 0 seis, las fuerzas competitivas (1995), o por otros agentes (Bueno 1996); hecho 
que, en el caso que nos ocupa, se produce a través de a implicación en la intervención de las entidades financieras y las instituciones públicas, tal como reflejamos en la figura $\mathrm{n}-1$ :

Figura 1

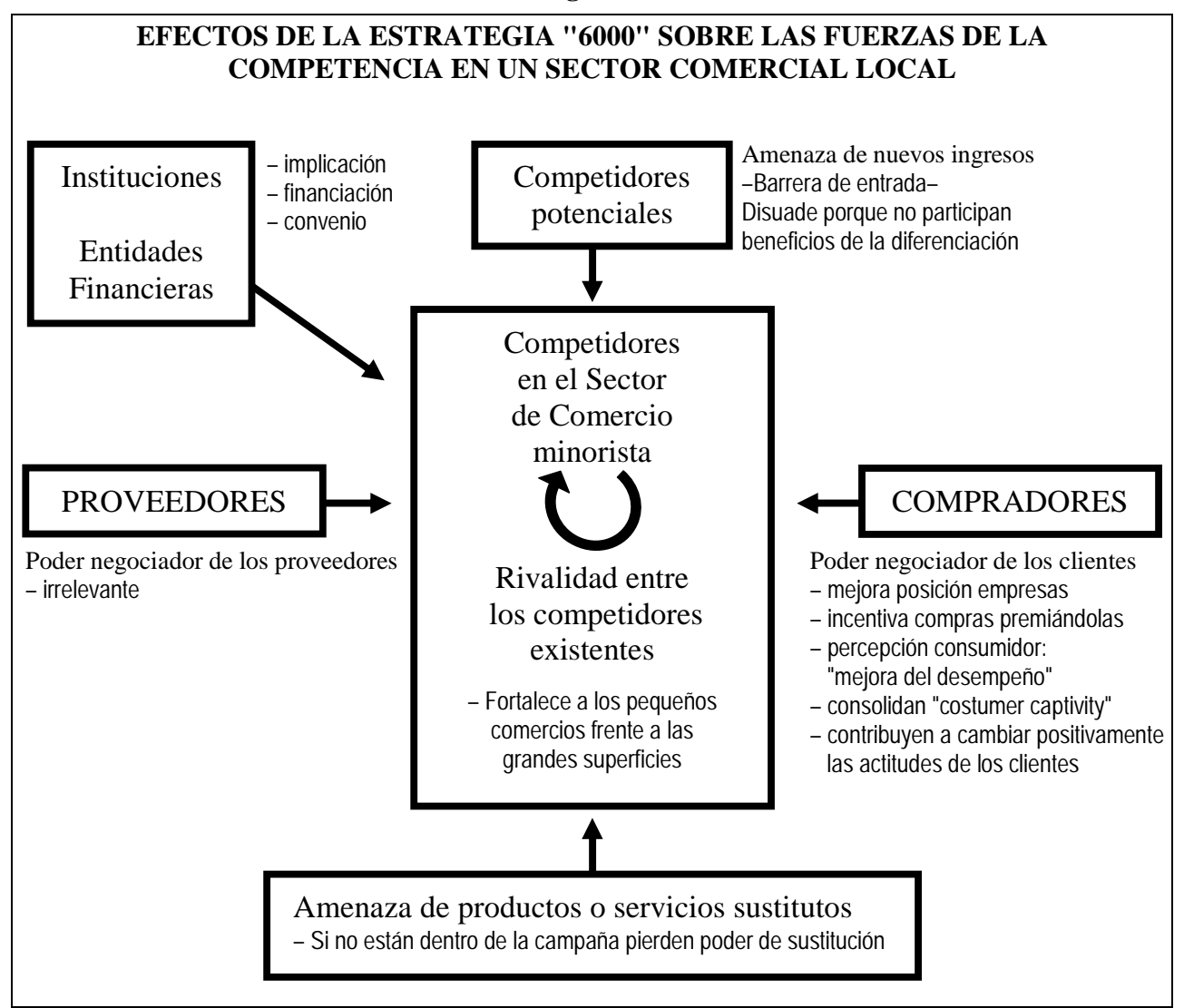

Fuente: Basado en Porter 1980 y Bueno 1996.

En definitiva, a tenor del análisis anterior y como hipótesis básicas de trabajo, que habremos de contrastar empíricamente mediante el estudio de campo, podemos formular:

Hipótesis 1: La Campaña 6000, en cuanto a estrategia empresarial cooperativa, mejora la posición competitiva de los pequeños comercios integrados en la estrategia frente a los no participantes estableciendo una diferenciación producto-servicio.

Accesoriamente y como Hipótesis adicionales contrastaremos: 
Hipótesis 2: Esta Estrategia de Diferenciación afecta sustancialmente a todas las fuerzas competitivas de un entorno local salvo a los proveedores y su poder de negociación que en nada se ve afectado.

Hipótesis 2.1: Respecto a la intensidad de la competencia actual refuerza las posiciones competitivas del pequeño comercio tradicional integrado en la práctica estratégica frente a los hipermercados y grandes superficies tradicionales.

Hipótesis 2.2: Respecto a la amenaza de entrada de nuevos competidores, actúa como barrera de entrada, disuadiéndoles de la entrada al no compartir los efectos de la diferenciación.

Hipótesis 2.3: Respecto a eventuales productos sustitutivos procedentes de otras firmas, pierden poder de penetración, al tener la desventaja en la diferenciación del proveedor.

Hipótesis 2.4: El efecto fundamental se produce sobre los clientes respecto a los que se mejora la capacidad negociadora sobre los mismos, de imponerles su producto sobre el aj eno en aspectos tales como:

a) Se incentiva la Compra, estimulando, ante la eventualidad del premio, al cliente.

b) El Cliente percibe una mejora en el desempeño por parte del comerciante.

c) Se fideliza durante el periodo la compra del cliente.

d) Se mejora por parte del cliente la percepción de la empresa trasmitiéndole valores de la misma como:

- Producto especializado

- Trato personalizado

- Compra de lo "propio y autóctono", lo de tu vecino, lo de tu amigo...

\section{EL CASO DE LA CAMPAÑA 6000 "BENAVENTE CIUDAD DE COMPRAS". SU ESTUDIO}

Como ya anticipamos, por su dimensión y abarcabilidad, hemos elegido la campaña de Benavente, una población castellanoleonesa enclavada en la provincia de Zamora, administrativamente cabeza de partido judicial con 45 kilómetros de término municipal y un 
censo de $18.609^{3}$ habitantes, que tienen un carácter eminentemente comercial como cabecera de un subárea comercial ${ }^{4}$, o una zona básica de comercio ${ }^{5}$, equidistante en torno a los 65 kilómetros de las capitales provinciales de León y de Zamora, y que abarca poblaciones del sur de la provincia de León y Norte de la de Zamora de en torno a los 50.000 habitantes, que afluyen fielmente para sus compras a la cabecera comarcal de Benavente, municipios rurales de pequeño tamaño cuya población oscila entre los 200 y los 3.500 habitantes. En las Navidades de 2004-5 se realiza por primera vez la descrita campaña 6000 bajo el lema. "Benavente, ciudad de Compras", que a continuación pasamos a estudiar.

\subsection{La situación fáctica. Los antecedentes}

En el término de Benavente, entre los años 1989 y 2005 se han asentado diez grandes superficies de 7 cadenas, con un total de superficie comercial de 7.800 metros cuadrados ${ }^{6}$, implantaciones que han experimentado una aceleración desde el año 2000 (en 1999 eran siete los establecimientos con 4.180 metros cuadrados $^{7}$ ). Ratio de superficie comercial elevadísima, que resultaría incomprensible de no tener en cuenta el carácter de Centro comercial comarcal de la ciudad. Siendo los siguientes en la actualidad:

- FROIZ, Avda. del Ferial, 57

- AlimerKa S. A., Avda. El Ferial, 15

- ALIMERKA S.A., Cañada de la Vizana, 10

- MERKA PRECIOS, Avda. El Ferial, 97

- MERKA PRECIOS, San Antón Viejo, 1

- MERCADONA, El Ferial, 99-101

- DIA S.A., Federico Silva, 8

- SUPERA S.A., Herreros, 53

- SUPERA S. A., Plaza Madera, 2 (desde 2005 EROSKI)

- GRUPO LIDL, Avda Federico Silva, 8

3 Ayuntamiento de Benavente, Padrón municipal de junio de 2005.

4 El Atlas Comercial de España (1994) establece para la provincia de Zamora un área comercial propia para la capital con tres cabeceras subáreas Benavente, Zamora y Puebla de Sanabria.

5 Plan Regional de Ámbito Sectorial de Equipamiento Comercial de Castilla y León (2005), Decreto 104/ 2005 de 29 de diciembre BOCyL no 251 de 30 de diciembre de 2005, artículo 19, 3.

y Urbanismo.

6 Datos proporcionados por el Ayuntamiento de Benavente-Concejalía de Obras

7 Asociación española de Centros Comerciales : www. aedecc.com. 
Respecto a estas grandes cadenas podemos establecer una ponderación de su superficie media en $780 \mathrm{~m}^{2}$, destacando MERCADONA y LIDL, por rebasar los $1.500 \mathrm{~m}^{2}$, y que, por ende, han precisado licencia especial conforme a la legislación de Castilla y León por rebasar estas dimensiones en poblaciones de entre 10.000 y 50.000 habitantes (R.D. 19 de diciembre de 2002).

Esta situación de hecho, en este espacio local, supone un caso especialmente agudo de concentración de superficie comercial, arrojando una densidad comercial de grandes superficies de $428 \mathrm{~m}^{2}$. por cada mil habitantes, ratio que coloca a la población entre las densidades medias más altas de España comparables a Madrid y Asturias ${ }^{8}$, situación que solo explica la dependencia comercial de una rica comarca agrícola de regadío, esta dependencia comercial corrige la densidad en $160 \mathrm{~m}^{2}$ por mil habitantes, cifra que, aunque es algo superior, es homologable al resto de Castilla y León.

\subsection{Los actores}

Aunque con un número notoriamente superior de establecimientos de pequeño comercio, que hay que fijarlos en $382^{9}$, integrados en la Federación de Comercio de Benavente, y por tanto participantes en la campaña "Benavente Ciudad de Compras" hay 118 establecimientos con una superficie media ponderada de $46 \mathrm{~m}^{2}$, lo que arroja una superficie comercial conjunta aproximada de $5.428 \mathrm{~m}^{2}$, es decir el $72 \%$ de las 10 grandes superficies con las que compiten. Siendo los siguientes:

Tabla 1

\begin{tabular}{|c|l|l|}
\hline $\begin{array}{l}\text { Establecimientos } \\
\text { Número }\end{array}$ & Ubicación & Sector Comercio \\
\hline 19 & Centro urbano y periferia 2 & TEXTIL \\
\hline 3 & Centro & DROGUERÍA-PERFUMERÍA \\
\hline 5 & Centro & JOYERÍAS \\
\hline
\end{tabular}

8 Datos de la Asociación Española de Centros Comerciales, con Madrid con 393 $\mathrm{m}^{2}$ por 1.000 habitantes y Asturias con 371 .

9 Padrón municipal de Actividades Económicas 2005. Ayuntamiento de Benavente.

10 Obtenidos los datos a partir de la relación de los 118 asociados participantes en la Campaña "Benavente-Ciudad de Compras" facilitados por CEOE-CEPYME-Benavente. 


\begin{tabular}{|c|c|c|}
\hline 5 & Centro & REGALOS \\
\hline 6 & Centro y 1 en periferia & LIBRERÍAS \\
\hline 6 & Centro & CALZADOS \\
\hline 4 & 3 centro y 1 periferia & ELECTRODOMÉSTICOS-ELECTRICIDAD \\
\hline 3 & Centro & DECORACIÓN \\
\hline 5 & Periferia & MOBILIARIO GENERAL- COCINAS \\
\hline 2 & Centro & INMOBILIARIAS \\
\hline 3 & Centro & MATERIALES CONSTRUCCIÓN \\
\hline 3 & Centro & ALIMENTACIÓN GENERAL \\
\hline 1 & Centro & ALIMENTACIÓN- PANADERÍAS \\
\hline 3 & 2 centro y 1 periferia & ALIMENTACIÓN- CARNICERÍAS \\
\hline 2 & Centro & ALIMENTACIÓN- PESCADERÍAS \\
\hline 2 & Centro & ALIMENTACIÓN- CONGELADOS \\
\hline 4 & Centro & PASTELERÍAS \\
\hline 1 & Centro & BODEGAS \\
\hline 2 & Centro & PELETERÍAS \\
\hline 4 & 3 centro y 1 periferia & ARTÍCULOS DEPORTIVOS \\
\hline 5 & 3 centro y 2 periferia & PELUQUERÍAS \\
\hline 1 & Centro & ÓPTICAS \\
\hline 2 & Centro & DISCOS-MÚSICA \\
\hline 3 & 2 centro y 1 periferia & FLORISTERÍAS \\
\hline 2 & Centro & JUGUETERÍAS \\
\hline 4 & Centro & FOTOGRAFÍA \\
\hline 2 & Centro & INFORMÁTICA \\
\hline 3 & Centro & TELEFONÍA \\
\hline 2 & Centro & PUBLICIDAD \\
\hline 4 & Periferia & COMERCIAL AGROPECUARIA \\
\hline 4 & Periferia & TALLERES-REPUESTOS \\
\hline 3 & Centro & OTROS \\
\hline
\end{tabular}

El predominio más evidente es el de moda: textil y calzado con 25 establecimientos entre ambas $(19+6)$, el $21,19 \%$ el sector alimentación y afines con 16 establecimientos, el 13,56\% y también con cifras relevantes joyería, regalos, librerías, en torno a 6 establecimientos cada uno, sobre un $5 \%$ cada uno de ellos y con una cierta relevancia de peluquerías, pastelerías, fotografía, talleres y comerciales agropecuarias, entre los 4-5 establecimientos asociados (en torno al 3,5\%). El resto de las actividades presenta una gran atomización. Actividades en la mayoría en las que incurren competitivamente las grandes superficies como ya reseñamos en el epígrafe 2 de este trabajo (González Bueno 2000). Estas 118 empresas constituían la relación de asociados y, por ende, participantes en la Campaña 6000. 


\subsection{La Campaña}

"Benavente, ciudad de Compras. Te damos 6000 euros porque somos tu comercio" se articula con la participación de los 118 comercios descritos que constituían la Asociación de Comercio de Benavente, que a su vez suscriben un convenio de colaboración y financiación con dos instituciones: Junta de Castilla y León (a través de su Consejería de Fomento) y Ayuntamiento de Benavente, dos asociaciones CEOE-CEPYME Benavente y la Cámara de Comercio de Zamora y una entidad financiera: Caja España (en el caso Zamora Caja Rural de Zamora). La duración de la campaña conforme a sus bases se establecía entre el 27 de noviembre y el 29 de diciembre, celebrándose el sorteo conclusivo el 3 de enero de 2005, conforme a 8 bases que se transcriben en la figura 2 .

El contexto en el que se desarrolló esta estrategia concreta fue en el de un plan estratégico más amplio y global, elaborado por los técnicos de CEOE-CEPYME: El Plan de revitalización del pequeño comercio, que establecía 5 áreas estratégicas de actuación: Promoción, animación comercial, formación, convenios comerciales y ayudas a la financiación, con un presupuesto total de 36.000 euros, de los que la campaña que nos ocupa se dotaría con 21.000 euros. 
Figura 2. Bases de la Campaña "Benavente, ciudad de compras"

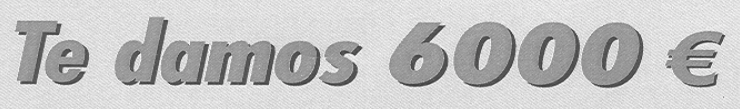
porque somos iv Comercio

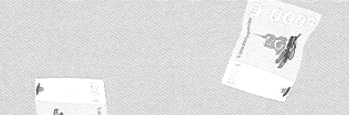

the
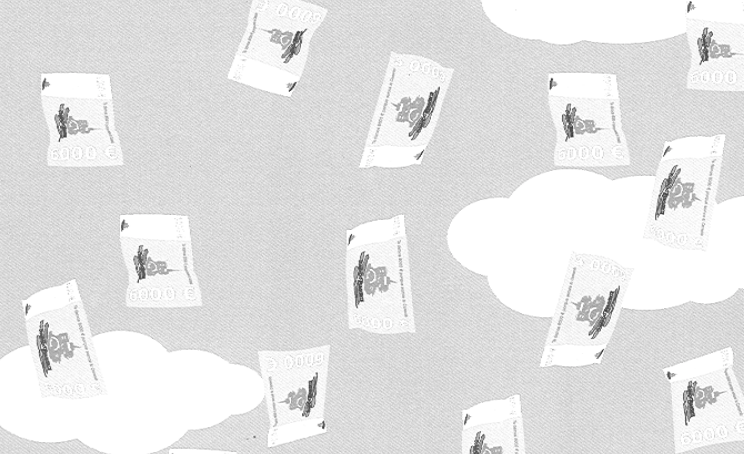
(t) ter. 数a (h)

12:-

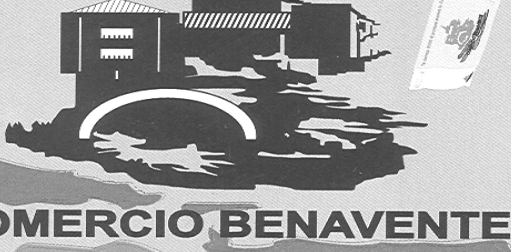

COMERCIO BENAVENTE

Benavente Ciudad de compras

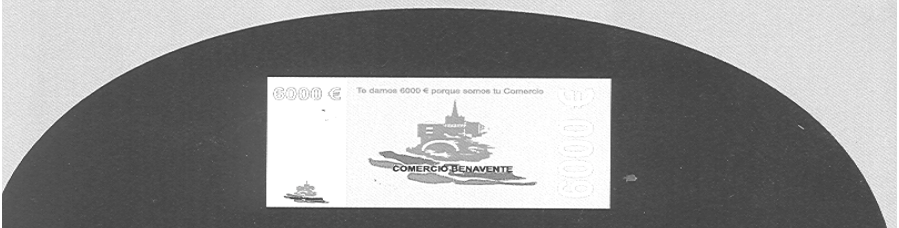

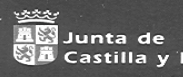

(i) Ayputamiento

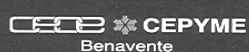
Caja España Cámarra 


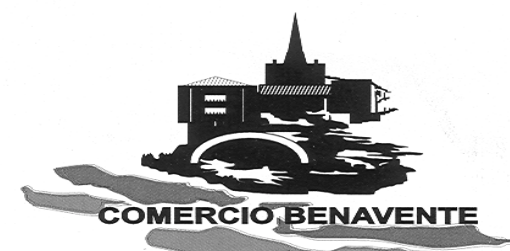

BASES

A partir del 25 de noviembre del 2004 , los comercios participantes en la Benavente, sita en C/ de los Carros, $37-2$. , para que se les facilite el material de dicha Campana consistente en. un cart, una banda para con los boletos de 100 números cada uno, numerados correlativamente.

Primera: Los boletos se entregarán por parte del comerciante a sus clientes de forma voluntaria en la cantidad que considere oportuna, atendiendo a las compras de sus clientes, durante el
periodo del 27 de noviembre al 29 de diciembre de 2004 .

Segunda: Todos los boletos para ser válidos deberán contener el nombre apellidos, teles urnas, existentes al efecto en cualquiera de las oficinas de Caja España en el municipio de Benavente, en el Ayuntamiento de

Tercera: Todos las boletos irán numerados correlativamente.

Cuarra: El día 3 de Enero del 2005 a las 12:00 h., en el lugar elegido
al efecto, se extraerán ante notario el boleto premiado y diez boletos suplentes.

Quinta: El ganador será avisado por teléfono ese mismo día, 3 de Enero del 2005, entre las 12:00 y las 16:00 $\mathrm{h}$. Para contactar con el premiluidos en el boleto premiado, con un máximo de dos números de teléfono por boleto, que en caso de no ser atendida personalmente después de esperar 15 tonos, se pasara inmediatamente a los suplentes por el mismo orden en que tonos La llamada podrá ser atendida por el propio premiado por alguna de las personas de su entorno, debiendo aceptar el premio personalmente el premiado o su cónyuge, hasta las aceptar el premio rechazarlo en el horario estabo de no realizará el mismo procedimiento para los suplentes, atendiendo al orden en que hayan sido extraídos.

Sexta: La persona ganadora no podrá delegar el premio, salva que concurrese una causa de fuerza mayor que debera acreditar que se constituya al efecto la que determine si existe fuerza mayor. De ser asi, el premiado podria delegar el premio exclusivamente en su cónyuge o ascendientes y descendiente

Séptima: No se podrán presentar al sorteo los titulares de los comercios

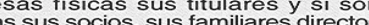
(cónyuge 0 ascendientes y descendientes en primer grado de cor canguras pudiendo participar con boletos obtenidos en otros establecimientos distintos del propio. Tampoco podran participar en el sorteo los empleados y miembros de las Juntas Directivas Comercio de Benavente, ni sus familiares directos (conyuge descendientes en primer grado de consanquinidad). En el caso de ser extraído un boleto correspondiente a alguna de las

El premio consistirá en SEIS MIL EUROS que deberá ser gastado por el ganador en los comercios participantes en la

1. Todas las compras se deberán realizar durante el día 4 de
Enero de 2005 en horario de comercio.

2. El ganador podrá elegir libremente para realizar sus compras entre

3. En caso de no gastar los 6.000 euros integramente en las

La persona ganadora tendrá a su disposición un vehiculo que le trasladará a los establecimientos elegidos para realizar sus compras.

5. El importe máximo que podrá gastar en cada comercio será
de 300 euros, excepto en uno de ellos, que podrá realizar que supere los límites establecidos en estas bases correrá a cargo del ganador.

6. El ganador está obligado a realizar compras nuevas, en
ningún caso se podran hacer pagos de deudas existentes con anterioridaden el comercio. 
3.4. El Estudio. Diseño de la investigación y Metodología

De forma breve describiremos el proceso de recogida de datos y análisis de la información.

a) Método de obtención de la información

El instrumento de obtención de información por parte de las empresas participantes es un cuestionario postal autoadministrado, en el que los encuestados contestan por escrito sin intervención directa de persona alguna relacionada con la investigación (Babbie 1995). El referido cuestionario estaba dirigido a los propietarios y directivos de las 118 empresas implicadas en la campaña y constaba de dos bloques:

I DATOS EMPRESARIALES. Referidos a:

- Actividad de la empresa

- Propiedad de la Empresa (Empresa familiar o no)

- Número de trabajadores, y

- Superficie del local comercial.

II ASPECTOS DE LA CAMPAÑA, como segundo bloque en el que se cuestionaba acerca de:

- Percepción de la misma por la empresa como estratégicamente positiva

- Percepción de la empresa de la presencia de grandes superficies como competencia perjudicial

- Percepción del incremento estacional de ventas navideñas

- Creencia de que una parte del incremento de ventas estacionales se debía a la campaña, y en cuyo caso en qué porcentaje

- Otros beneficios o efectos positivos de la Campaña

b) Universo de estudio

Los datos se obtuvieron a partir de la elaboración del cuestionario descrito sobre la población total de las 118 empresas (los 118 asociados y participantes en la campaña), ascendiendo el número de respuestas válidas a 76 , conforme a la siguiente ficha técnica: 
Tabla 2: Ficha técnica

\begin{tabular}{|r|l|}
\hline \multicolumn{2}{|c|}{ FICHA TÉCNICA DEL PROCESO } \\
\hline Unidad de Análisis & La Empresa \\
\hline Universo & $\begin{array}{l}\text { Pequeñas empresas comerciales radicadas en Benavente } \\
\text { participantes en la Campaña 2004-5 "Benavente Ciudad } \\
\text { de Compras" }\end{array}$ \\
\hline Ámbito geográfico & Local- Ciudad de Benavente \\
\hline Tamaño muestral & 118 encuestas con 76 respuestas válidas- 64,41\% \\
\hline Trabajo de Campo & Meses de Julio y Agosto de 2005 \\
\hline Cuestionario & Estructurado en dos bloques con preguntas cerradas \\
\hline
\end{tabular}

Tras la recepción de datos no fue necesario eliminar ningún formulario de los 76 recibidos, por ajustarse a los parámetros subjetivos la formulación de respuestas y contener todos ellos información sustancial.

\section{c) Variables. Su medición}

La estructura de la propiedad de las empresas fue estimada mediante una variable dicotómica ( $1=$ empresa familiar; $0=$ empresa no familiar).

La antigüedad mediante el año de su fundación.

Los datos de la campaña, planteados dicotómicamente de modo cerrado como percepciones positivas o negativas se valoraron igualmente ( $1=\mathrm{si}-\mathrm{v} . \mathrm{gr}$. considera positiva la campaña-; $0=$ no).

En lo que respecta a la ponderación del incremento de las ventas debido a la actuación cooperativa se midió mediante una pregunta de escala en términos porcentuales (\%). Restando como pregunta abierta "Otros efectos positivos de la campaña" con espacio para 3 items, que se ponderó en términos de frecuencia de respuesta.

d) Resultados

1) Estructura de la propiedad

De las 76 encuestas útiles valoradas en 59 casos se trataba de empresas familiares (el $77,63 \%$ ) y de entre ellas 32 , es decir el $54,24 \%$ de las empresas familiares datan de más de una generación, existiendo un caso significativo, Almacenes Imperio del sector textil que se remonta a 3 generaciones. 
Figura 3

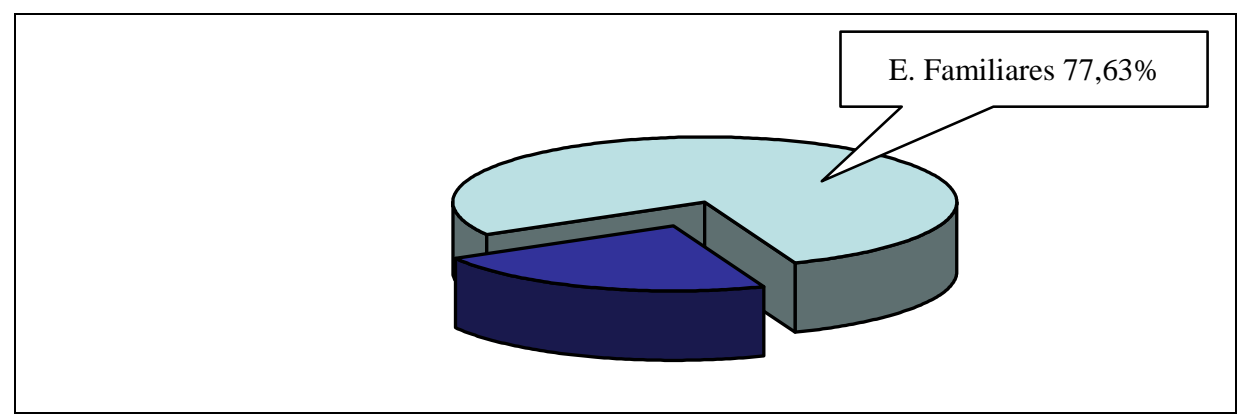

2) Forma jurídica

Del total de 76 empresas encuestadas 49 constituyen empresa individual, y 27 tienen forma societaria, con la siguiente distribución: 17 sociedades limitadas, 4 comunidades de bienes, 3 cooperativas (de trabajo asociado) y 3 S.A.

Tabla 3

\begin{tabular}{|l|c|c|}
\hline \multicolumn{1}{|c|}{ Forma jurídica } & Número & Porcentaje \\
\hline Empresas individuales & 49 & $64,47 \%$ \\
\hline Sociedades Limitadas S.L. & 17 & $22,37 \%$ \\
\hline Comunidades de Bienes & 4 & $5,26 \%$ \\
\hline Cooperativas & 3 & $3,95 \%$ \\
\hline Sociedades Anónimas & 3 & $3,95 \%$ \\
\hline
\end{tabular}

En cuanto al número de operarios se trata en todo caso de microempresas, la mayoría regentada por su titular, con, a lo sumo, uno, dos o tres empleados, salvo el caso de almacenes Imperio y Talleres Ramón con 4 y 6 empleados respectivamente.

Una vez definida la estructura de propiedad, el predominante carácter familiar de las empresas y su escaso número de empleados, estamos en condiciones de enjuiciar esta estrategia cooperativa a la luz de los resultados empíricos.

3) La Campaña 6000. Discusión de los Resultados

Los resultados de la encuesta coinciden mayoritariamente en que se trata de una campaña contextuada en una época en que 
se produce un incremento estacional de las Ventas, 68 del total de 76 encuestados (el 89,50\%). Un número aún más elevado coincide en que el establecimiento de supermercados y grandes superficies (73 encuestados), el $96,10 \%$ ha perjudicado notablemente el comercio tradicional, de forma que, sin duda, hay que inferir que este ha sido un elemento fuertemente motivador para la articulación de estos modelos estratégicos que apuestan por la cooperación.

Igualmente, aunque de modo más matizado, una mayoría significativa percibía que parte del incremento de ventas se debe a la realización de esta actuación concertada (46 encuestas), el $60,5 \%$ y el porcentaje de incremento en las ventas por efecto directo de la campaña, que fijan, calculado como promedio, es del $8,23 \%$ Finalmente respecto a la pregunta abierta con tres ítems de los otros efectos positivos que produce la campaña son los siguientes ordenados por mayor frecuencia de respuesta:

1) Mejora de la imagen Comercial del colectivo

2) Aproximación al consumidor (Identificación con la zona, "compras lo tuyo a los tuyos", trato directo...)

3) Excelencia (mayor valor de su producto, personalización, especialización...).

En cuanto a la constatación de las hipótesis teóricas formuladas de partida, entendemos que de la interpretación conjunta de los resultados anteriores queda plenamente justificada la Hipótesis 1: que esta actuación estratégica mejora la posición competitiva de sus actores como consecuencia de la mejora de percepción del servicio (el 60,5\%) traduciéndose en un incremento de ventas del $8,23 \%$

Respecto a la hipótesis 2, relativa a al incidencia en las otras fuerzas competitivas, los resultados han de tomarse con más cautela, a falta de de otras constataciones empíricas futuras, y aunque es previsible conjeturar una nula o escasa influencia sobre el poder negociador de los proveedores, esta no ha quedado acreditada (subhipótesis 2.1), otro tanto ocurre con las subhipótesis 2.2 y 2.3 (amenazas de entrada y productos sustitutivos, que precisarían de estudios empíricos complementarios. 
Sí queda, en cambio, plenamente acreditada la hipótesis 2.4, en tanto que la estrategia, dirigida fundamentalmente a clientes, incrementa el poder negociador de esta pequeñas empresas familiares sobre los mismos, incentivando sus compras ante la eventualidad del premio, el incremento de las ventas del $8,23 \%$ como efecto directo de la estrategia, así lo avala, al igual que la percepción de la mejora del desempeño por parte del cliente con la percepción de compra de lo autóctono, el trato personalizado, o la idea de comercio especializado y próximo que provee satisfacción, incluso emocional, al cliente que siente y percibe que adquiere lo próximo, lo autóctono, lo de su vecino; emociones todas que entran en la esfera de motivaciones de pertenencia y estima, ya clásicamente descritas por Maslow (1943). Aunque sin duda esta última conclusión habría de ser afinada y matizada por encuestas y análisis empíricos futuros dirigidos a los clientes.

\section{CONCLUSIÓN}

La implantación en poblaciones de pequeño y medio tamaño de hipermercados y grandes superficies comerciales ha supuesto una muy sustantiva alteración de la competencia por parte del pequeño comercio tradicional, básicamente constituido por empresas de carácter familiar. Estas firmas han visto, en poco más de una década, drásticamente reducidas sus cuotas de mercado, padeciendo una suerte de darwinismo comercial.

Frente a esta situación, en diversos entornos locales de Castilla y León, el pequeño comercio ha reaccionado estratégicamente, configurado, al socaire y amparo de las asociaciones y federaciones empresariales, imaginativas estrategias defensivas como reacción. Estrategias de carácter cooperativo que muestran sensibilidad hacia el entorno sociocultural en que se desarrollan (Bigné, Moliner y Sánchez 2005). Tales actuaciones, por razones obvias de dimensión, tamaño y capacidad económica, se han orientado hacia una estrategia promocional, contextuada en un fenómeno de asociacionismo espacial, que ha incidido en la diferenciación, exaltando el valor competitivo de su tamaño, especialización y proximidad, constituyendo un notable ejemplo de congruencia entre la estrategia y la cultura en la que se insertan.

Entre estas estrategias, sobresale una, la que hemos estudiado bajo la denominación de "Campaña 6000", que se ha implantado 
estacionalmente en Navidad con éxito, en distintas poblaciones de Castilla y León, fundamentándose en un merchandising común, que ha evidenciado sus fortalezas, la fortaleza de "los pequeños" y que nos sugiere nuevas perspectivas, reflexiones y enfoques estratégicos en ámbitos locales.

\section{BIBLIOGRAFÍA}

ARAgón SÁnCHeZ, A. y I. RuBio BAÑón (2005) "Factores asociados al éxito cooperativo de las PYMES industriales en España", Universia Business Review, 8, 4은 Trimestre, pp. 38-51.

Asociación Española de Centros Comerciales: www. aedecc.com.

Atlas Comercial de España (1994) Banco Central Hispano, Madrid.

BARNEY, J . (1991) "Firm Resources and Sustained Competitive Advantage", J ournal of Management, 17, pp. 99-120.

BIGNÉ, J .E. (2003) Promoción comercial: Un enfoque integrado. Mac Graw Hill.

-; Moliner, M.A.; SÁnCHEZ, J . (2005) "El enfoque cultural de la Orientación al mercado y los resultados empresariales. El efecto moderador del entorno", Cuadernos de Economía y Dirección de Empresas, 23, pp. 139-164.

Bueno Campos, E. (1996) Dirección Estratégica de la Empresa. Metodología, Técnicas y Casos. Madrid: Pirámide (5aㅡ).

BurnetT, J .J . (1997) Promoción: Conceptos y estrategias. Mac Graw Hill.

CECALE (Confederación de Empresarios de Castilla y León): www.ceca le.es

CEOE-CEPYME- Zamora: www. ceoecepymeza.org.

CONFAES (Confederación Abulense de Empresarios): www.avilaempresa rial.com.

DíEZ dE CASTRO, J . (1994) Distribución comercial. Mac Graw Hill.

GalBRAITH, J.K. (1967) The new Industrial State. Boston: Houghton Miffin C. Versión española: El Nuevo Estado Industrial. Barcelona: Ariel, 1980.

GonZÁlez BenITO, O. (2000) "Competencia espacial intraurbana de las cadenas líderes de hipermercados en España: Modelización explicativa 
en el marco de la estrategia de localización", Revista española de Investigación de Marketing ESIC, vol. 4, no1, pp. 131-141.

GRANT, R.M. (1995) Contemporary Strategy Analysis: Concepts, techniques, applications. Cambridge: Blackwell Publishers. Versión española: Madrid: Civitas, 1996.

Greenwald, B. y J. Kahn (2005a) Competition Demystified: A Radically Simplified Approach to Business Strategy. New York: Portfolio.

- y - (2005b) "All Strategy is Local", Harvard Business Review, September, pp. 95-104.

HARRIGAN, K.R. (1999) "Estrategias del final de partida para sectores en decadencia", Porter, M.E.: Ser Competitivo. Nuevas aportaciones y conclusiones. Bilbao: Deusto, pp. 165-123.

HAX, A.C. y N.S. MAJ LUF (1997) Estrategias para el Liderazgo Competitivo. Buenos Aires: Granica.

MASLOW, A. (1943) "A Theory of Human Motivation", Psychological Review, 50, pp 370-396.

NAVAS LóPEZ, J .E. y L. A. GuerRas Martín (2002) La Dirección Estratégica de la Empresa. Teoría y Aplicaciones. Madrid: Thompson-Civitas (3a).

Plan Regional de Ámbito Sectorial de Equipamiento Comercial de Castilla y León (2005) Decreto 104/ 2005 de 29 de diciembre BOCyL no 251 de 30 de diciembre de 2005.

PORTER, M.E. (1980) Competitive Strategy. New York: The Free Press. Versión española: México: CECSA, 1982.

- (1987) The competitive advantage. Versión española: Ventaja competitive (creación y sostenimiento de un desempeño superior). México: CECSA, 2002.

- (1990) The Competitive Advantage of Nations. New York: The Free Press. Versión española: La ventaja competitiva de las Naciones. Barcelona: Plaza \& J anés, 1991.

SCHUMACHER, E.F. (1973) Small is beautiful. Versión española: Lo pequeño es hermoso. Madrid: H. Blume ediciones, 1982.

ThOMPSON, A. y A.J. StRICKLAND (2001) Administración Estratégica. México: $\operatorname{CECSA}\left(11^{a}\right)$. 